\title{
Circuit
}

Musiques contemporaines

\section{Faux et usage de faux, réponse aux mélodistes indépendants} Réponse à Anne Lauber

\section{Response to Anne Lauber}

\section{Robert Normandeau}

Volume 8, numéro 1, 1997

Autoportraits. Montréal, l’après 1967

URI : https://id.erudit.org/iderudit/902194ar

DOI : https://doi.org/10.7202/902194ar

Aller au sommaire du numéro

Éditeur(s)

Les Presses de l'Université de Montréal

ISSN

1183-1693 (imprimé)

1488-9692 (numérique)

Découvrir la revue

Citer ce document

Normandeau, R. (1997). Faux et usage de faux, réponse aux mélodistes indépendants : réponse à Anne Lauber. Circuit, 8(1), 77-80.

https://doi.org/10.7202/902194ar
Résumé de l'article

À la suite de la réédition, dans le volume VII, $\mathrm{n}^{0} 1$ de CIRCUIT, de l'entrevue d'Anne Lauber et des Mélodistes indépendants où il avait été mis en cause, le compositeur de musique acousmatique fait une mise au point. 


\title{
Faux et usage de faux, réponse aux mélodistes indépendants
}

\author{
Robert Normandeau
}

Madame Anne Lauber,

Si je m'adresse à vous aujourd'hui plutôt qu'au groupe des Mélodistes indépendants, c'est que, dans l'article signé Claude Gingras paru dans La Presse du 13 mai $1995^{(1)}$, vous me preniez directement à partie en faisant référence à notre travail comme membres d'un jury du ministère de la Culture au cours de la saison 1993-1994. Le retard avec lequel je vous réponds aujourd'hui n'est redevable qu'à mon absence du pays du 10 mai au 13 juin $1995 \mathrm{ce}$ qui m'avait empêché de vous répondre dans la Presse. Ce n'est donc qu'à mon retour que j'ai pris connaissance de ce que vous écriviez sur mon manque de conscience professionnelle, accusations qu'il convient de dénoncer ici avec la plus grande vigueur.

Premièrement, vous commettez une faute de déontologie grave en révélant publiquement le contenu des délibérations des membres d'un jury. Par le fait même, vous avouez publiquement que votre signature n'est d'aucune valeur puisque, vous l'avez sans doute oublié, en quittant cette réunion, vous avez signé un document vous engageant à la confidentialité des débats qui y ont eu lieu.

Deuxièmement, il convient ici d'éclairer le public quant au fonctionnement interne des jurys afin de dissiper l'un des préjugés les plus communs et les plus détestables dont vous vous faites le malheureux héraut. Les jurys lautant ceux du Québec que ceux d'Ottawa) sont formés de professionnels du milieu choisis pour leurs compétences respectives et provenant de disciplines différentes. Ainsi, au sein du jury dont nous avons fait partie, vous représentiez la composition instrumentale, Jean Saulnier, pianiste classique, représentait l'interprétation et moi la composition électroacoustique et la musique actuelle. C'est grâce à cette représentativité et au fait que les jurys sont constamment renouvelés qu'il existe une grande diversité dans l'attribution des bourses au sein de nos organismes subventionnaires. De plus, le principe de fonctionnement généralement adopté pour ce genre de jury est celui du consensus. Sur un jury formé de trois personnes seulement, il est mathématiquement impossible qu'une bourse soit
(1) Cet article a été repris dans le numéro de CIRCUIT (1996) consacré au débat public qui a fait suite à un éditorial de Lise Bissonnette publié dans Le Devoir du 3 octobre 1994. Ce débat portait sur la responsabilité des créateurs face à la rupture avec le public. (N.d.R.) 
attribuée sans le consentement de tous ses membres. Comme moi, vous avez approuvé toutes les décisions qui y ont été prises.

Troisièmement, ce que vous dites quant au fonctionnement de notre jury est bourré de contradictions et d'erreurs grossières. Vous dites: "J'ai demandé aux deux autres membres du jury pourquoi ils s'obstinaient dans leur refus. Ils m'ont répondu : "Pour une question d'esthétique." Pour eux la musique de Rachel Laurin c'était trop tonal, trop mélodique. ». Et plus loin : "Mais Saulnier n'était ni pour, ni contre. " Alors, Mme Lauber, il faut vous décider, soit les autres membres du jury refusent, soit ils ne sont ni pour, ni contre! Quand la mauvaise foi vous guide... Et plus loin : "C'est la fonctionnaire du ministère qui a fait pencher la balance. "Autre préjugé tenace (décidément, cela vous en fait beaucoup à porter) : les fonctionnaires n'ont pas droit de vote sur les jurys ef mon expérience comme membre de jury lquatre jurys à Ottawa et quatre jurys à Québec) m'a toujours mis en présence de responsables tentant au contraire de faire prévaloir aux membres les aspects négligés par ceux-ci, soucieux qu'ils sont de répartir le plus équitablement possible les maigres fonds qu'ils ont à leur disposition. C'est donc ajouter l'insulte à l'injure quand, en plus de trahir votre engagement de confidentialité, vous médisez d'une fonctionnaire qui avait eu l'élégance de vous inviter. D'autant plus que celle-ci, tout au long des délibérations, a pris une grande quantité de notes au sujet de toutes les demandes et ces notes, assemblées afin de répondre aux candidats qui veulent connaître les raisons invoquées par le jury pour approuver ou désapprouver leurs demandes, vous les avez lues, approuvées et vous les avez signées. Pourtant, dans l'entrevue avec Claude Gingras, vous dites: "Ce que je trouve dégoûtant, c'est que lorsque Rachel a téléphoné pour connaître la vraie raison du refus, ils n'ont jamais voulu la lui dire. Il y a de la manipulation. "Avez-vous donc la mémoire si courte? C'était il y a plus d'un an, il est vrai...

Quatrièmement, et c'est là que le bât blesse personnellement, vous dites: " Il y avait Robert Normandeau. C'est un électroacousticien et il a voté pour tous ses petits copains. "Je rappelle pour mémoire que le jury dont nous faisions partie distribuait des bourses aux musiciens de musique de concert, interprètes et compositeurs confondus. Au cours des trois réunions de ce jury (mai et septembre 1993, janvier 1994), nous avons distribué 31 bourses pour un montant global de $151638 \$$ (tous ces chiffres sont publics et facilement vérifiables). Ces bourses ont été réparties de la façon suivante : $103688 \$$ à 21 interprètes et $37600 \$$ à 6 compositeurs. Á tous ces heureux récipiendaires, s'ajoutent 4 compositeurs de musique électroacoustique qui ont reçu $10350 \$$, ce qui correspond à 7 pour cent de la somme globale. Désolé, les copains, de n'avoir pas fait mieux! Pour le reste, surtout en ce qui a trait aux interprètes, $j$ 'ai fait comme n'importe quel professionnel consciencieux et j'ai écouté les cassettes, j'ai lu les dossiers que l'on nous remettait toujours à l'avance et j'ai écouté les avis de mes pairs (que voulez-vous, je ne suis pas un spécialiste du hautbois baroque...). 
Au fond, ce qu'il y a de plus inquiétant dans votre façon de dire les choses, c'est que vous reproduisez exactement ce que vous dénoncez. Quand vous dites : «Il y a de la manipulation » d'un côté et que de l'autre vous affirmiez "Que Schoenberg ait été mis à la porte du conservatoire explique bien des choses... ", omettant de signaler au passage qu'il l'ait été parce qu'il était juif, comment appelez-vous cela? Quand vous reprochez aux autres membres du jury de s'être prononcé " pour une question d'esthétique » et que par ailleurs vous affirmiez que « 95 pour cent du temps, l'électroacoustique, c'est du bruit confus ", ne croyez-vous pas qu'il est plus qu'équitable que de temps en temps un compositeur comme moi siège sur des jurys ? Sinon, comment la musique électroacoustique québécoise aurait-elle acquise ses lettres de noblesse, elle dont les représentants ne cessent de récolter des prix tant sur le plan national qu'international ? Comment cette musique électroacoustique, sans des qualités aussi évidentes qu'indéniables, aurait-elle pu convaincre les différents jurys qui ont permis à empreintes DIGITALes, la compagnie de disque québécoise spécialisée en électroacoustique, de devenir la plus importante compagnie privée dans le domaine? Non, madame, je ne crois pas que nos institutions ni nos organismes de diffusion soient à ce point déficients. Allez donc voir ailleurs en France, en Belgique, en Angleterre, en Allemagne, en Suisse même - et vous comprendrez mieux de quoi je parle. Vous comprendrez également que ce n'est pas parce qu'on encourage les nôtres qu'on le fait au détriment des autres membres de la profession et que les choix se font sur une base strictement professionnelle.

Montréal, le 26 juin 1995

GINGRAS, C. et les mélodistes indépendants (1996), « À bas la musique contemporaine ! » in « Ruptures ? \#, CIRCUIT, revue nord-américaine de musique du $x x^{e}$ siècle, vol. VII, nº 1, pp. 55-60. 


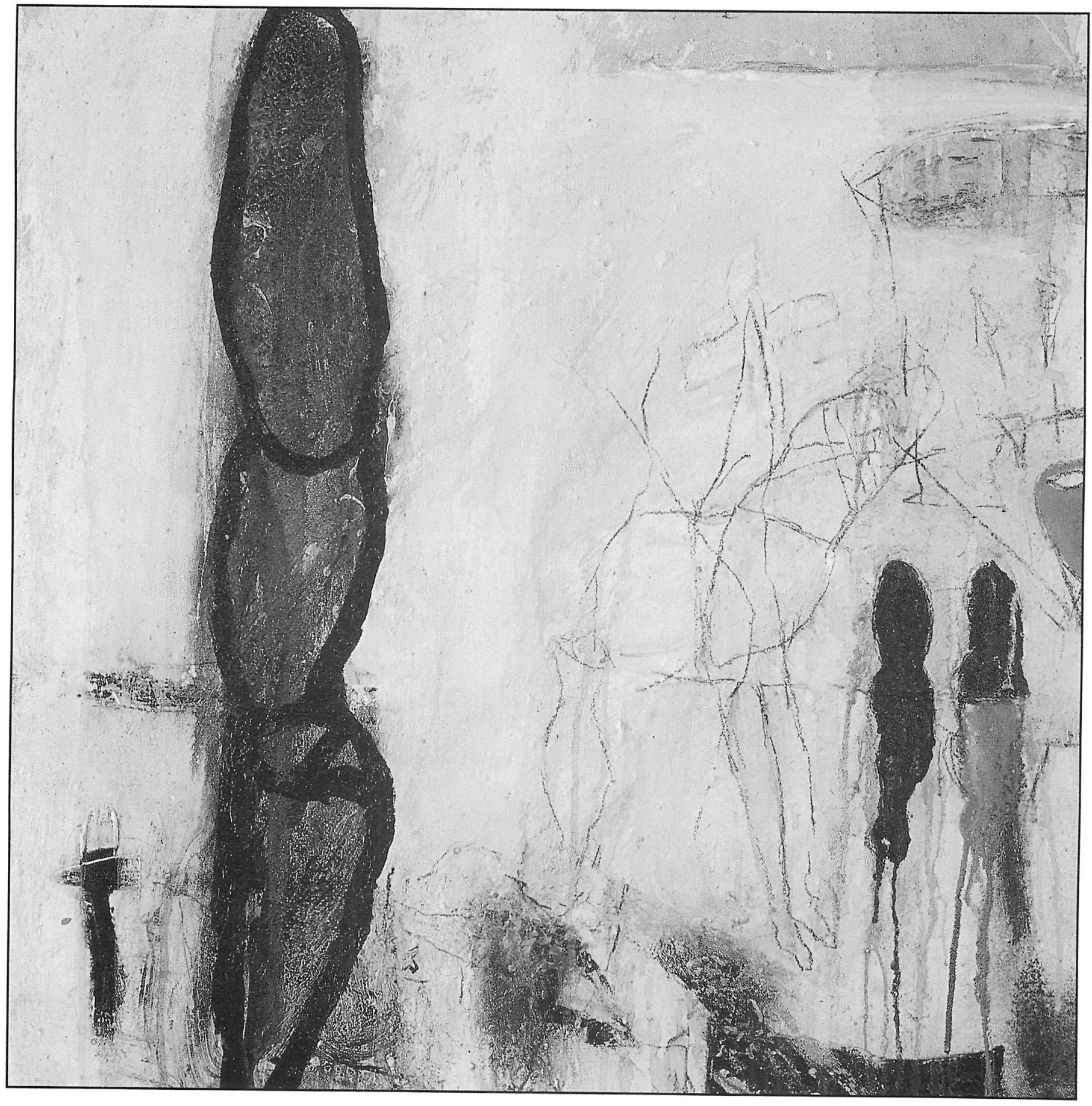

